\title{
Light-Emitting Diode-Based Pulsed Illumination Source for Continuous, Cycle-Resolved Spray Measurements
}

\author{
Markus Mühlthaler*1, Franz-Josef Nagler ${ }^{1}$, Georg Wachtmeister ${ }^{1}$ \\ ${ }^{1}$ Department of Mechanical Engineering, Technical University of Munich, Munich, Germany \\ *Corresponding author email : muehlthaler@lvk.mw.tum.de
}

\begin{abstract}
This paper presents a modular, high-powered light emitting diode (LED)-based illumination source to be used for spray measurements of the liquid phase via Mie scattering. The recent availability of consumer-grade LED arrays with average rated radiant powers of more than $100 \mathrm{~W}$ have enabled lower-cost designs than were possible with the previous laser illuminants. Flash tubes suffer from a non-constant rate of emission intensity and a fixed discharge time, which can be a restricting factor, especially with multiple injections. The narrow spectral distribution of LEDs, with a full width at half maximum of $17 \mathrm{~nm}$, aids in distinguishing the measurement signal from unwanted background noise.

Using frame-synchronous LED pulse generation at repetition rates of $100 \mathrm{kHz}$, it is possible to record both arbitrarily spaced multiple injections as well as consecutive working cycles, for example in internal combustion engines. Pulse durations are currently variable in the range 1$78 \mu \mathrm{s}$.

Luminous flux is boosted by driving the light source at a significantly higher current and voltage as compared to the continuously rated specifications, which results in a high temporal resolution, as exposure times as low as one $\mu$ s provide ample recorded intensities.

Benchmarks based on a commercial xenon flash tube and laser diode demonstrate the design's versatility along with first applications in an optically accessibly research engine.
\end{abstract}

\section{Keywords}

Pulsed LED, Mie scattering, dual-fuel, high-speed recording

\section{Introduction}

Over the course of a few years, readily available light emitting diodes (LED) for consumers have experienced an increase in radiant power from just a few to several hundreds of watts per chip-on-board (COB) device. This increase, combined with minimal procurement costs, a long service life, and very little maintenance compared to conventional sources such as flash tubes, laser diodes or pulsed laser systems, make them a viable alternative source of illumination. They can be operated in both continuous and pulsed mode, with the latter resulting in increased luminous flux from overdriving the LEDs in current and voltage for low duty cycles. Another advantage of pulsed operation is the negligible pulse-to-pulse variation in emitted intensity and spatial distribution. LEDs can be switched at flexible, arbitrary frequencies or operating patterns with little dead time and are subject to fewer external constraints than flash tubes or pulsed laser systems. The incoherent light typically has a spectral distribution of several tens of nanometers, which is broad enough to prevent the kind of speckle patterns formed in laser illumination, yet still narrow enough to distinguish the scattered signal using an optical band pass, in order to suppress unwanted background noise. Due to their large numerical aperture and surface area on COB devices, they are especially suitable for volumetric illumination setups. If collimated light is required, additional efforts are necessary. The literature reports LED applications in the context of schlieren imaging [1], Mie scattering [2,3], particle image velocimetry [4-6] and shadowgraphy in combination with multiplexed illumination for high frame rates [7]. In contrast, conventional illumination sources 
for fuel sprays in internal combustion engines are based on flash tubes [8], laser diodes [9] or pulsed lasers [10].

In the present study, selected LEDs are characterized by their rise time, intensity and spectral emission behavior when operated with pulsed drive currents. The results highlight their benefits and limitations compared to other illumination sources. The authors discuss the details of the implemented circuit design and integrated functions. The article concludes with an outline of example applications of the resulting device in a side-scatter arrangement.

Table 1 - Specifications of the investigated LEDs and the illumination sources used for comparison.

\begin{tabular}{c|c|c|c|c}
\hline Type & $\begin{array}{c}\text { Forward } \\
\text { current [A] }\end{array}$ & Power [W] & $\Phi_{\mathrm{v}}[\mathrm{Im}]$ & $\begin{array}{c}\text { Wavelength } \\
\text { center [nm] }\end{array}$ \\
\hline LED $_{\text {green }}$ LC100G-C45 & 3.5 & $100 \mathrm{cw}$ & 7.500 & 522 \\
LED blue JH100B14G45-Z1C $_{\text {Laser diode (LD) Cavilux Smart }}$ & 3.5 & $100 \mathrm{cw}$ & 1.700 & 462 \\
Flash tube (FT), Hensel VH3-6000 & & $\begin{array}{c}360 \mathrm{pulsed} \\
6000 \mathrm{~J} / \text { pulse }\end{array}$ & & $\begin{array}{c}685 \\
\text { Broadband }\end{array}$
\end{tabular}

\section{Characteristics of pulsed LED operation with high drive current}

Table 1 lists the specifications of the LEDs examined in this study along with the illumination sources used for comparison. Both are of the chip-on-board type, in which an array of small LEDs is mounted on a printed circuit board to form the individual package. The literature highlights the potential of increasing luminous flux by abandoning continuous operating parameters when moving over to pulsed operation with a high current combined with a low duty cycle [5,6,11-13]. All measurements are based on the circuit developed in-house.

Pulsed operation and temporal trigger response. Figure 1 relates the increased emitted intensity of pulsed operation at $50 \mathrm{kHz}$ with higher-than-rated currents to the nominal operating point. The green variant achieved 5.3 more intensity at 9.9 times the rated current, while the blue variant attained 7 times more intensity at 15.7 times the rated current at $\mathrm{T}_{p}=8 \mu \mathrm{s}$. Due to the relatively low duty cycles, no noticeable damage or degradation occurred in the emitters.
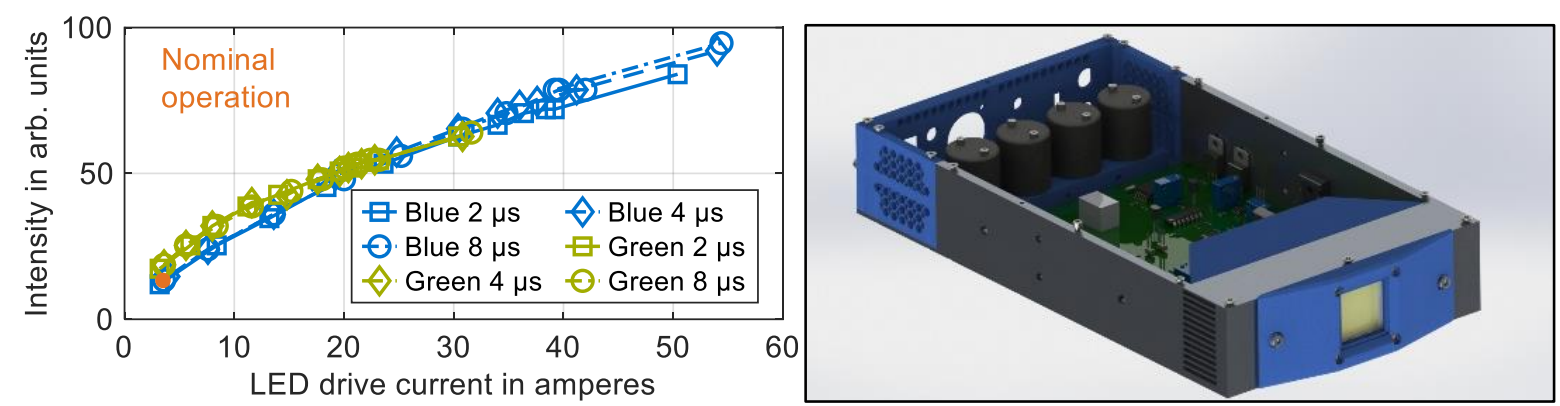

Figure 1. Left: Detectable intensity vs. LED drive current for the selected blue and green LED variants (JH100B14G45-Z1C, LC100G-C45), operating with $50 \mathrm{kHz}$ current pulses of varying durations from $\mathrm{T}_{\mathrm{p}}=2$ to 8 $\mu \mathrm{s}$, measured on a photodiode (SFH203P). Right: CAD model of the prototype without logic printed circuit board. 

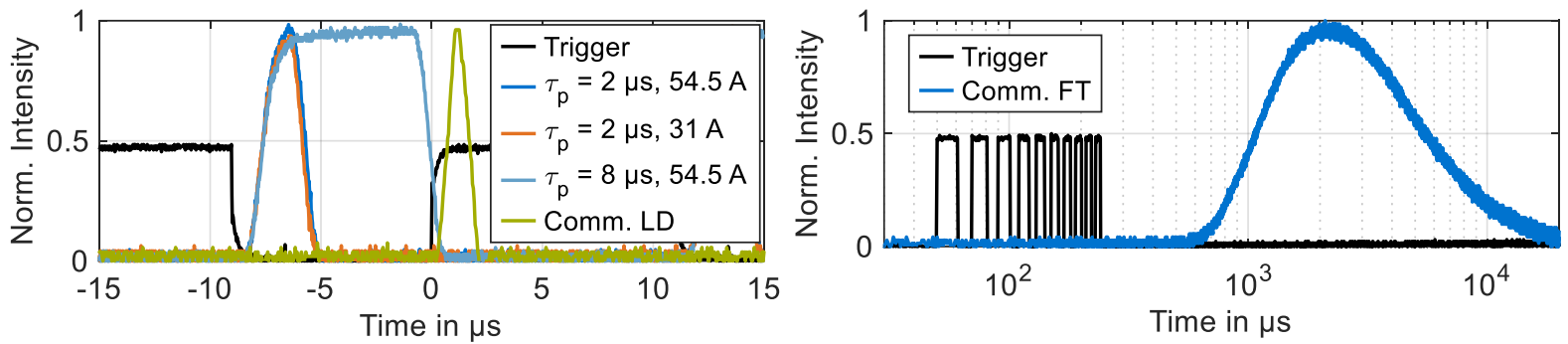

Figure 2. Left: Oscilloscope trace of the normalized intensity measured on a photodiode (SFH203P) in response

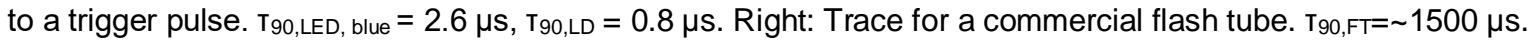

The slightly larger detected intensity for pulses longer than $T_{p}=2 \mu s$ is understood with the oscilloscope trace in Figure 2. The graph on the left shows the recorded intensity by a photodiode in response to a pulsed $50 \mathrm{kHz}$ trigger input. As the signal in the photodiode directly correlates to the driver current of the LED, the visible offset was verified with a current clamp. A constant rising flank with a rise time up to $90 \%$ total intensity T90,LED $=2.6 \mu \mathrm{s}$, beginning with the trigger signal, was observed with different pulse durations and current levels for the LED. In comparison, the rise time for the commercial laser diode was T90,LD $=0.8$ $\mu \mathrm{s}$. The graph on the right measures the light emission for a commercial xenon flash tube in response to an identical trigger input. A significantly prolonged rise time T90,FT $_{9} 1500 \mu \mathrm{s}$ is observed, while the overall emission time is close to $20 \mathrm{~ms}$ and not user-changeable. There is no individual reaction to subsequent triggers. On the contrary, the long emission is continuous and limits the experiment's timeframe, as a recharge time of several seconds is required after the flash. However, the laser diode enables variable pulse durations at small duty cycles - this particular model offers pulse durations of $10 \mathrm{~ns}$ at $30 \mathrm{kHz}$.

The LED, however, allows a wider variability to enable the active light emission to be adjusted to the current experiment. Also, the light intensity varies constantly with the temporal evolution of the flash, while in the case of the LED, the intensity reaches a plateau after the initial current rise. This aids the experiment, as smaller gradients are introduced due to the illumination, which are troublesome when, for instance, threshold levels are applied to distinguish between a spray and background noise.

Spectral properties. Compared to the xenon flash tube, the LED has a narrow spectral intensity distribution. The full width at half maximum for the blue LED is close to $17 \mathrm{~nm}$, see Figure 3 , and thus well suited to spectral filtering. This reduces the amount of unwanted background noise from the experiment in the detected signal, for instance, when the injection process in an internal combustion engine is ongoing while the flame is already propagating. While the FWHM of the aforementioned commercial laser diodes is still narrower at $4.52 \mathrm{~nm}$, the LED does not suffer from speckle originating from the laser diodes' narrow FWHM and coherent emission. For a tailored, narrow band pass filter, it is necessary to consider the spectral peakshift of the LED emissions. If only datasheet values were taken into account, most of the emission would be lost in pulsed operation with a high drive current, as shown in Figure 3. Besides the shift, a slight broadening of the FWHM from 17 to $21 \mathrm{~nm}$ is observed for high currents. Both effects depend on the junction material present, $[14,15]$. With the exception of a detector change to a charge-coupled device (pco.1600 monochrome), measurements were obtained with the setup described in [16]. 

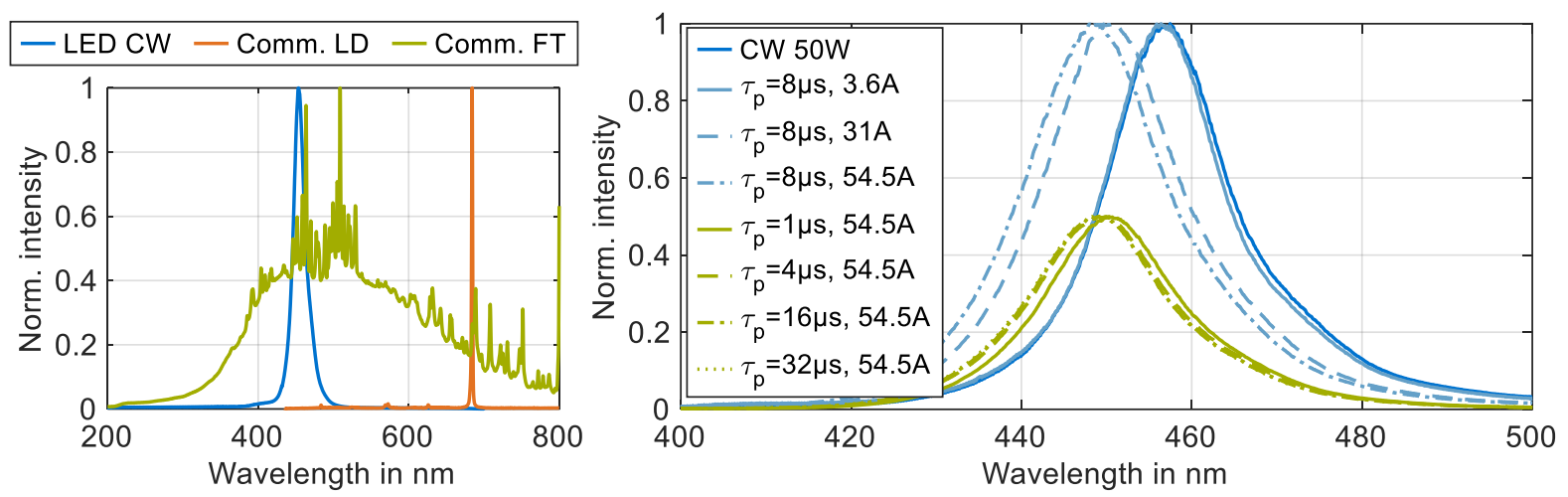

Figure 3. Left: Broadband spectral distributions for a blue and a generic white LED compared to a commercial flash tube. Right: Spectral peak shift for the blue LED resulting from pulsed operation and variation of pulse durations. The variation in pulse duration $\mathrm{T}_{\mathrm{p}}$ is scaled to 0.5 intensity to enable easier differentiation.

\section{Device Layout and Electrical Design}

Packaging and software functions. The authors' goal was to provide a compact, rugged and inexpensive package, which is capable of handling multiple measurement tasks, incorporating the selected LED and using easily available components. The LED's footprint limited the package's height in order to enable stacking in one dimension. Lenses for collimating and shaping the output can be mounted on the emitter side. As rising package temperatures reduces both luminous flux and service life, an active cooling system and heatsink designed after [17] are incorporated to increase stability and reduce heat input.

The software supports a master/slave configuration and the devices can be connected in a daisy chain formation with the $\mathrm{I} 2 \mathrm{C}$ bus, if multiple illumination angles or increased volume are required. Current illumination parameters, such as pulse length, power level or arbitrary triggering sequences primed by the first transistor-transistor logic high input (such as: drop every $\mathrm{n}^{\text {th }}$ trigger, start after or end after $\mathrm{x}$ triggers, pause for $\mathrm{y}$ triggers or time span), can be configured via a USB interface, and settings are retained in the microcontroller's non-volatile memory. An Atmel Atmega 328PU mounted on an Arduino Nano development board is responsible for overall system tasks, for example controlling the main capacitor voltage or heat sink temperature. The layout is divided into a logic board and the power stages driving the LED. Pulsed and continuous operation mode with constant current can be selected.

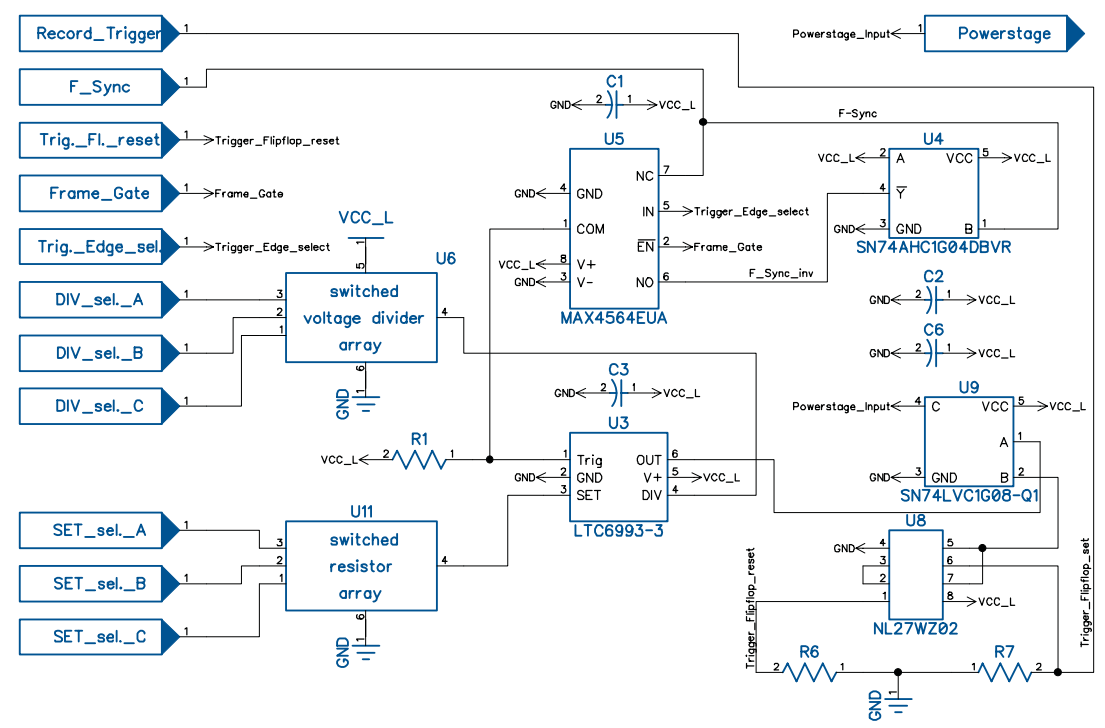

Figure 4. Schematic diagram of the logic circuit, formed around the LTC6993-3 one-shot pulse generator. 
Logic circuit. The use of discrete logic enables good timing accuracy and fast trigger responses, resulting in high operating frequencies without the need for a costly FPGA or microcontrollers with high clock speeds. The main inputs of this logic component are a frame dependent signal, the so called F-sync, and a second signal indicating the start of the actual frame sequence recorded by the camera, called Record_Trigger, see Figure 4. First, the Fsync is duplicated and inverted (U4). Depending on the level of the Trig-_Edge_sel signal, either the F-sync or the inverted signal is passed on and interacts with the Frame_Gate signal in an AND gate (U5). Then the resulting signal is fed into the timing chip LTC 6993-3, which acts as a one-shot pulse generator. The pulse duration results from the DIV and SET inputs, fed from a switchable voltage divider and resistor arrays switched by the microcontroller. The second trigger signal Record_Trigger acts on the SET input of an RS flip-flop(U8). Record_Trigger is only high for a short time and indicates the beginning of the actual recorded sequence. The flip-flop latches and remains in this state until Trigger_Fl_reset is set to high after the user-selected timespan. Only while the flip-flop is latched isthe output signal of LTC6993-3 able to pass through the final AND gate (U9) and reach the power stage.

Power stage. The previously conditioned and gated trigger signal is then fed into the dualinverting, high-speed MOSFET driver U12 (MC34151D), shown in Figure 5. Its associated output drives the gate of the N-channel MOSFET Q2 (IRFP150). Q2 is the main switching element in the circuit. It is specified with a maximum drain-to-source voltage of $100 \mathrm{~V}$ and a maximum peak current of $160 \mathrm{~A}$. Its gate input capacitance of $2800 \mathrm{pF}$ is charged via R20 and discharged via R21-D3. R20 limits the charging current to prevent damage to U12 from high short-circuit currents or unwanted feedback currents from the coupled drain-gate capacitance during switching. R21 and D3 enable faster discharge of the gate and thus a faster falling edge of the current driving the LED. Furthermore, D1 and D2 are connected to the gate, acting as a clamping circuit to partially turn on Q2 in the event of potential spikes above 82V and thus limiting the drain-source voltage. The resulting drain-source current is limited only by R23, the internal resistance of the LED, the drain-source resistance of Q2 and the internal resistance of C1. C1 consists of four low equivalent series resistance electrolytic capacitors of $2200 \mu \mathrm{F}$ each connected in parallel. This not only creates a favorable form factor for the device, but also further reduces the internal resistance of $\mathrm{C} 1$, enabling high discharge currents.

The voltage that $\mathrm{C} 1$ is charged to depends on the selected power level. Based on the general supply voltage of $24 \mathrm{~V}$, this adaptation can be efficiently performed using a DC-DC converter with a boost converter topology. In the present device, a commercial DC-DC converter module was modified to enable its output voltage to be set digitally between $30 \mathrm{~V}$ and $80 \mathrm{~V}$. Figure 6 illustrates this modification and the resulting response curve.

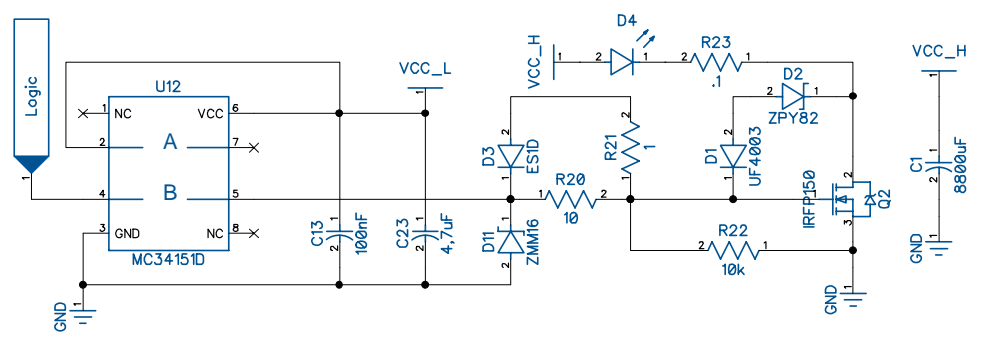

Figure 5. Power stage for driving the LED (D4) in pulsed operation using a MOSFET driver IC (U12) and an Nchannel MOSFET (Q2); energy is stored in the main capacitor (C1). 

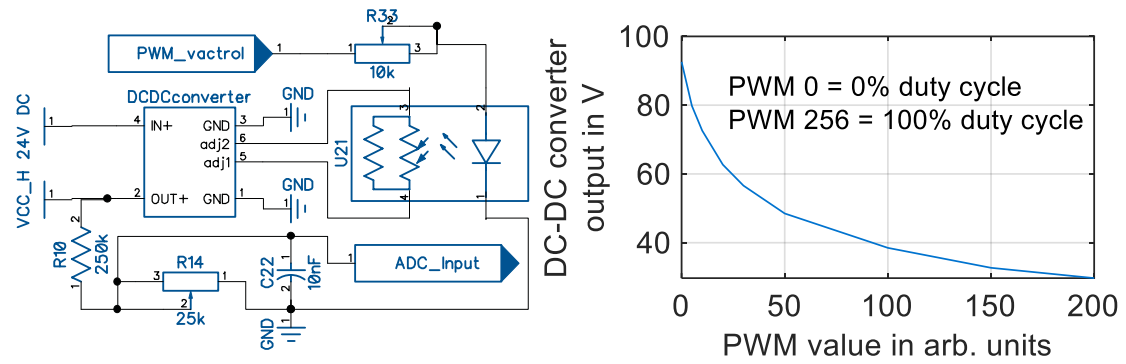

Figure 6. Left: Modified feedback path of the commercial DC-DC converter including a PWM-driven vactrol in place of a standard voltage adjustment potentiometer. Right: Output voltage of the modified DC-DC converter for driving the vactrol (U21) with an 8-bit PWM signal.

The module used has an adjustable output voltage of between $12 \mathrm{~V}$ and $80 \mathrm{~V}$, as well as an adjustable current limitation of up to $12 \mathrm{~A}$. It has an operating frequency of $150 \mathrm{kHz}$ and is equipped with short-circuit protection. Due to the lack of suitable digital potentiometers, current and voltage were set using a vactrol developed in-house. It consists of a micro-controlled LED as a light source and a light dependent resistor, which are optically coupled in a closed housing. The vactrol acts as a galvanically isolated adjustable resistor, whose operating voltage depends solely on the maximum voltage of the light dependent resistor. A pre-control value is calculated by linear interpolation of the response curve. To compensate for temperature drift, the output voltage is also measured via the ADC and the setpoint adjusted accordingly. A discretization-related deviation of $\pm 1 \mathrm{~V}$ is present at $80 \mathrm{~V}$.

\section{Example applications and performance characterization}

Figure 7 on the left presents two images of consecutive working cycles taken through the piston window of an optically accessible single-cylinder engine based on the bowditch principle, with port fuel injection of a natural gas/hydrogen mixture ignited via a pilot-injection of poly oxymethylene dimethyl ethers at 600 rotations per minute.

The exposure time of $85 \mu \mathrm{s}$ was necessary to gather sufficient counts of the weak natural luminosity of the combusting gas, while $T_{p, L E D}$ was set to $2 \mu \mathrm{s}$. The images show the second injection per cycle, while the weak signal from the flame propagation ignited by the first injection is already ongoing across most of the chamber,

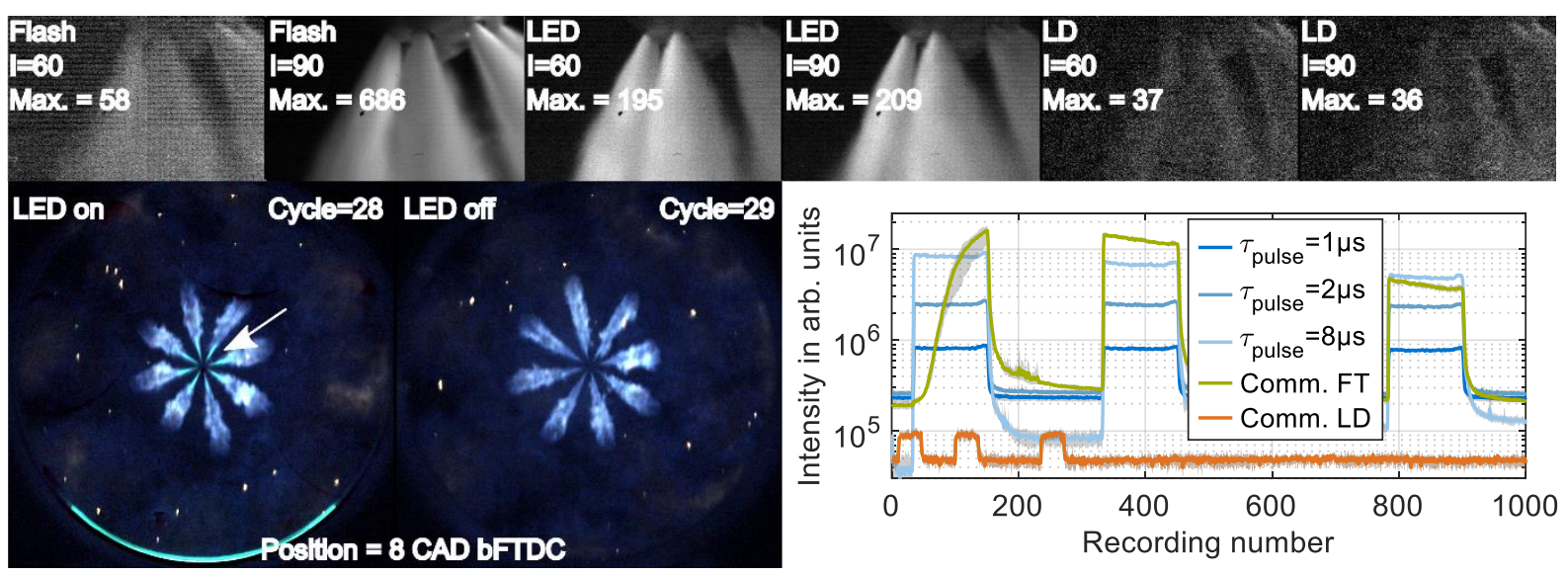

Figure 7. Top: Raw data for the plot on the right, at two time steps including maximum intensity. Left: Example recording of two consecutive fired cycles taken with a high-speed RGB camera, showing the second injection per cycle. No additional optical band pass filter was installed. $\mathrm{T}_{\mathrm{p}, \mathrm{LED}}=2 \mu \mathrm{s}$, camera exposure time $=85 \mu \mathrm{s}$, $\mathrm{f}=5.6$. Right: Comparison of the intensity evolution of the signals over time for three consecutive injections with the LED and the commercial samples, relayed via a long distance microscope (Questar QM-100 LD, $\mathrm{f}=20.1$ ). The laser diode was limited to $30 \mathrm{kHz}$, while all other devices recorded at $100 \mathrm{kHz}$, both storing 1000 frames. 
visible as a slight blue hue with occasional orange spots due to insufficient mixture preparation. The green LED is primed for alternate cycles, in order to isolate the visualized liquid core. Cycle 29 directly follows cycle 28 and the image corresponds to the same crank angle position. Despite an ongoing injection, the ignition has already begun, dominated by a blue flame. Even without the addition of optical band pass filters, the Mie scattered signal is distinguishable from the ignition of the injected liquid in cycle 28.

The graph on the right illustrates the temporal evolution of a series of 1000 images taken at frequencies of $100 \mathrm{kHz}$ for the flash tube and LED, and $30 \mathrm{kHz}$ for the laser diode. The sum of all pixels within a region of interest central to the spray's expansion is calculated for each image. Three injections are conducted within a timeframe of $10 \mathrm{~ms}$ with an individual duration of $1 \mathrm{~ms}$. The camera exposure time either follows the source pulse duration or is fixed at $1 \mu \mathrm{s}$ for the laser diode and flash lamp. Each curve represents the mean of five injection events, with the envelope plotted in grey. While the laser diode's fast response and rise time enable the entire event to be captured, the limited frequency diminishes the temporal resolution. The flash tube suffers due to the long response time and is unable to capture the whole event, degrading significantly in intensity over the injections, resulting in the need for post-processing to detect threshold levels for data extraction. This is also evident in the top row of Figure 7 , where a large gradient in intensity between recording number 60 and 90 for the flash tube, spanning an order of magnitude. The LED captures the event fully and attains sufficient signal intensities at $T_{p}=1 \mu \mathrm{s}$. Switching to an active $T_{p}=2 \mu$ s resulted in ample signal levels while limiting the motion blur present in the event without a relevant gradient in intensity. If higher pulse durations are required, the current amount of stored energy in combination with the high duty cycle of $80 \%$ are not sufficient to uphold the current continuously. In the given example, a diminished intensity is observed at the given frequency for $T_{p} \geq 8 \mu \mathrm{s}$.

\section{Conclusions}

In this study, a modular illumination source for scattering experiments was developed and benchmarked as an alternative to commercial solutions. The device is based on framesynchronous LED pulses. It increased the continuously rated luminous flux up to factor 7 by overdriving with a high current and voltage in pulsed operation. The operating parameters were verified up to a repetition rate of $100 \mathrm{kHz}$ and provided ample signal levels in the given experiments at pulse durations as low as $1 \mu \mathrm{s}$, while the peak signal intensity was reached after $2.6 \mu \mathrm{s}$.

Its advantage over commercial solutions is its ability to react to arbitrary trigger sequences of varying length, repetition and in-between delays, .e.g. from one working cycle to the next in an internal combustion engine or at cutouts within a single working cycle, with nearly constant luminous intensity throughout the sequence. The narrow spectral distribution enables the measurement signal to be distinguished spectrally form unwanted background noise. As the applied LEDs are available in a range of colors, they can be tailored to different experimental conditions.

At a cost of roughly $200 €$ per unit the device is competitively priced for volumetric illumination applications, where there is no necessity for planar sheet imaging or a structured light illumination approach. 


\section{Contributions}

Mühlthaler M. - Conceptualization, Methodology, Investigation, Data Curation, Writing Original Draft, Review \& Editing, Visualization, Project administration; Nagler F. Conceptualization, Methodology, Software, Investigation, Writing - Original Draft, Visualization; Wachtmeister G. - Funding acquisition, Supervision, Writing - Review.

\section{Acknowledgments}

The authors wish to thank Ulrich Tetzner for the fruitful discussions on matters relating to electrical engineering.

\begin{tabular}{lll}
\multicolumn{2}{l}{ Nomenclature } & \\
COB Chip on board & LED & Light emitting diode \\
CW Continuous wave & MOSFET & Metal-oxide-semiconductor FET \\
FWHM Full width at half maximum [nm] & PWM & Pulse width modulation \\
FT Flash tube & $\mathrm{T}_{\mathrm{p}}$ & Pulse duration [s] \\
f\# $\quad$ F-stop & $\mathrm{T}_{90}$ & $90 \%$ Intensity response time [s] \\
LD Laser diode & $\Phi_{\mathrm{v}}$ & Luminous flux [Im]
\end{tabular}

\section{References}

[1] Willert C. E., Mitchell D. M., and Soria J., 2012 Exp Fluids, 53(2), pp. 413-421.

[2] Zhang A., Montanaro A., Allocca L., Naber J., and Lee S.-Y., 2014 SAE Int. J. Engines, 7(2), pp. 1034-1043.

[3] Montanaro A., Migliaccio M., Allocca L., Fraioli V., Lee S.-Y., Zhang A., and Naber J., 2014 SAE Technical Paper 2014-01-1406.

[4] Nasibov H., Balaban E., Kholmatov A., and Nasibov A., 2014 Flow Measurement and Instrumentation, 37, pp. 12-28.

[5] Willert C., Moessner S., and Klinner J., 25.-28.08.2009 8th International Symposium on Particle Image Velocimetry.

[6] Willert C., Stasicki B., Klinner J., and Moessner S., 2010 Meas. Sci. Technol., 21(7), p. 75402.

[7] Kornienko V., Kristensson E., Ehn A., Fourriere A., and Berrocal E., 2020 Scientific reports, 10(1), p. 16650.

[8] Wloka J. A., Pötsch C., and Wachtmeister G., 05.-07.09.2011 24th European Conference on Liquid Atomization and Spray Systems.

[9] Lonn S., Matamis A., Tuner M., Richter M., and Andersson O., 04.-06.04.2017. WCX ${ }^{\mathrm{TM}}$ 17: SAE World Congress Experience.

[10] Purwar H., Wang H., Tang M., Idlahcen S., Rozé C., Blaisot J.-B., Godin T., and Hideur A., 2015 Optics express, 23(26), pp. 33396-33407.

[11] Willert C., Freitag S., and Hassa C., 08.-10.09.2008 22nd European Conference on Liquid Atomization and Spray Systems (ILASS).

[12] Buchmann N. A., Willert C. E., and Soria J., 2012 Exp Fluids, 53(5), pp. 1545-1560.

[13] Stasicki B., Hiller W. J., and Meier G., 1984 tm - Technisches Messen, 51(6).

[14] Manninen P., and Orreveteläinen P., 2007 Appl. Phys. Lett., 91(18), p. 181121.

[15] Ju Z. G., Tan S. T., Zhang Z.-H., Ji Y., Kyaw Z., Dikme Y., Sun X. W., and Demir H. V., 2012 Appl. Phys. Lett., 100(12), p. 123503.

[16] Mühlthaler M., Blochum S., Stadler A., Härtl M., Wachtmeister G., MIYAMOTO A., and Sauerland H., 13.-15.04.2021. WCX ${ }^{\mathrm{TM}}$ 21: SAE World Congress Experience:

[17] Seo B.-S., Lee K.-J., Yang J.-K., Cho Y. S., and Park D.-H., 2012 Transactions on Electrical and Electronic Materials, 13(6), pp. 292-296. 